\title{
PRODUTIVIDADE E QUALIDADE DOS FRUTOS DA LARANJEIRA 'PÊRA' CLONE IAC EM 16 PORTA-ENXERTOS NA REGIÃO DE BEBEDOURO-SP ${ }^{1}$
}

\author{
EDUARDO SANCHES STUCHI², LUIZ CARLOS DONADIO ${ }^{3}$, OTÁVIO RICARDO SEMPIONATO \\ DILERMANDO PERECIN ${ }^{4}$
}

\begin{abstract}
RESUMO - Avaliou-se a influência de 16 porta-enxertos na produtividade, nas características físicas e químicas (sólidos solúveis totais- ${ }^{\circ}$ Brix; acidez; ratio; porcentagem de suco; índice tecnológico e tamanho dos frutos) dos frutos da laranjeira 'Pêra' [Citrus sinensis (L.) Osbeck] e na incidência e severidade da clorose variegada dos citros (CVC). O plantio do experimento foi realizado em julho de 1993, com espaçamento de 6,0 m entre linhas e 3,5 m entre plantas (476 plantas/ha). O experimento foi conduzido sem irrigação. O delineamento experimental foi em blocos ao acaso, duas plantas por parcela, três repetições e 16 tratamentos, constituídos pelas seguintes cultivares porta-enxertos: tangerineira 'Sun Chu Sha Kat' (Citrus reticulata Blanco), tangerineira 'Pectinífera' ( $C$. reticulata), 'Shekwasha' ( $C$. depressa Hayata), tangerineira 'Pectinífera/Shekwasha' $(C$. depressa Hayata), tangerineira 'Batangas' (C. reticulata), tangerineira 'Oneco' (C. reticulata), citrangor [citrange (Poncirus trifoliata Raf. x C. sinensis) x C. sinensis], citrandarin [C.sunki hort. Ex Tanaka) x Poncirus trifoliata (L.) Raf. cv. English, tangerineira 'Sunki' (C. sunki), tangerineira 'Suen-Kat' (C. sunki), tangerineira 'Nasnaran' ( $C$. amblycarpa Ochse), tangerineira 'Venezuela' $(C$. reticulata), tangerineira Heen Naran ( . lycopersicaeformis hort. ex Tan. ), limoeiro 'Cravo' (C. limonia Osbeck) x tangerineira 'Cleópatra' (C. reshni hort ex Tanaka), limoeiro 'Cravo' $(C$. limonia $)$, tangerineira 'Cleópatra' $(C$. reshni). A intensidade da clorose variegada dos citros variou em função dos porta-enxertos e não se relacionou com a produção de frutos até a quarta safra. Os porta-enxertos estudados, com exceção da tangerineira Nasnaran, proporcionaram qualidade e produções iniciais de frutos similares aos do limoeiro 'Cravo'.
\end{abstract}

Termos para indexação: Citrus sinensis, clorose variegada dos citros.

\section{YIELD AND FRUIT QUALITY OF 'PÊRA' SWEET ORANGE CLONE IAC ON 16 ROOTSTOCKS IN BEBEDOURO REGION, STATE OF SÃO PAULO, BRAZIL}

\begin{abstract}
An experiment was set up in July 1993, at Bebedouro city, São Paulo state, Brazil, aiming to evaluate the rootstock influence on the yield and several physical and chemical fruit characteristics such as fruit size, total soluble solids, acidity, ratio juice content and technological index of 'Pera' IAC sweet orange [Citrus sinensis (L.) Osbeck], in a high inoculum pressure area of citrus variegated chlorosis (CVC). The spacement was 6.0 $\mathrm{m}$ between rows and $3.5 \mathrm{~m}$ between trees. The experimental design used was randomized blocks, with three replications and two trees per plot. The rootstocks tested were 'Sun Chu Sha Kat' mandarin (Citrus reticulata), 'Pectinífera' (C. reticulata), 'Shekwasha' (C. depressa Hayata), 'Pectinífera/ Shekwasha' (C. depressa Hayata), 'Batangas' (C. reticulata), 'Oneco' (C. reticulata), citrangor [citrange (Poncirus trifoliata Raf. x C. sinensis) x C. sinensis], citrandarin (C. sunki hort. ex Tanaka x Poncirus trifoliata L. Raf. cv. English), 'Sunki' (C. sunki), 'Suen-Kat' (C. sunki), Nasnaran (C. amblycarpa Ochse), 'Venezuela' mandarin (C. reticulata), 'Heen Naran' mandarin (C. lycopersicaeformis), 'Cravo' (C. limonia Osbeck) x 'Cleopatra' (C. reshni hort ex Tanaka), 'Cravo' (C. limonia), 'Cleopatra' (C. reshni). CVC intensity was different due to rootstocks effects and not related with yield until the fourth year of production. With the exception of the Nasnaran mandarin the rootstocks induced initial fruit production and quality similar to 'Cravo'.
\end{abstract}

Index Terms: Citrus sinensis, citrus variegated chlorosis.

Atualmente, o limoeiro ‘Cravo’ é o principal porta-enxerto da citricultura paulista ( $80 \%$ das plantas estão enxertadas sobre ele, embora seja intolerante ao declínio (Pompeu Júnior, 2001) e à morte súbita dos citros (Müller et al., 2002). Em dezembro de 2000, o Fundecitrus registrou que $28,2 \%$ das mudas da laranjeira 'Pêra' estavam enxertadas sobre limoeiro 'Cravo', e as mudas de 'Pêra' representavam $31,1 \%$ das existentes nos viveiros (Salva, 2001). A laranjeira 'Pêra' é a principal variedade cultivada no Estado de São Paulo. Em 2000, existiam 92,7 milhões de árvores plantadas desta cultivar, representando $37,8 \%$ das plantas cítricas do Estado (Pompeu Júnior, 2001). O limoeiro ‘Cravo’ é classificado como indutor de boa qualidade aos frutos das variedades enxertadas (Pompeu Júnior, 1991). Porém outros porta-enxertos, como as tangerineiras 'Cleópatra' e 'Sunki', o citrumelo 'Swingle', a laranjeira 'caipira' e o citrangeiro 'Troyer' e o trifoliata, além de serem tolerantes ao declínio dos citros, à gomose e à morte súbita dos citros, podem proporcionar qualidade superior à do limoeiro 'Cravo'. A qualidade dos frutos é influenciada pela clorose variegada dos citros (CVC) (Laranjeira \& Palazzo, 1999). Alguns dos porta-enxertos tolerantes ao declínio (tangerineiras 'Cleópatra' e 'Sunki', citrumelo 'Swingle') e à morte súbita dos citros (tangerineiras 'Cleópatra' e 'Sunki', citrumelo 'Swingle' e trifoliata) têm algumas desvantagens em relação ao limoeiro ‘Cravo': a 'Cleópatra' tem a entrada em produção mais tardia; trifoliata, 'Cleópatra', 'Swingle e
'Sunki' apresentam, em ordem decrescente, maior suscetibilidade ao estresse hídrico; 'Swingle' e trifoliata são incompatíveis com 'Pêra', e a 'Sunki' é mais suscetível à gomose (Pompeu Júnior, 1991; Müller et al., 2002). Laranjeira et al. (2001) relataram não haver influência dos portaenxertos na expressão da CVC quando consideradas a incidência em plantas e ramos, a proporção de ramos infectados e a concentração relativa de bactéria em folhas. Stuchi et al. (1998) também reportaram a não-interferência dos porta-enxertos na intensidade da CVC quando consideradas a incidência e a severidade em conjunto através de um índice de doença calculado com base nas duas primeiras. O objetivo deste trabalho foi avaliar a influência de 16 porta-enxertos na Produtividade e na qualidade dos frutos de laranjeira 'Pêra' cultivada na região de Bebedouro (SP), em condições de alta pressão de inóculo da clorose variegada dos citros.

$\mathrm{O}$ experimento foi conduzido na Estação Experimental de Citricultura de Bebedouro (E.E.C.B.), município de Bebedouro-SP, em um solo classificado como Latossolo Vermelho-Escuro, epieutrófico, endoálico, A moderado, textura argilosa. O clima da região é subtropical com inverno moderado e seco, verão quente e chuvoso, com temperatura média de $23,5^{\circ} \mathrm{C}$; a precipitação anual média é de $1.522 \mathrm{~mm}$. O plantio do experimento foi realizado em julho de 1993, sob espaçamento de $6,0 \mathrm{~m}$ entre linhas e 3,5 m entre plantas (476 plantas/ha). O experimento foi

\footnotetext{
${ }^{1}$ (Trabalho 177/2003). Recebido: 21/11/2003. Aceito para publicação: 25/06/2004. Parcialmente financiado pelo Fundecitrus.

${ }^{2}$ Pesquisador da Embrapa Mandioca e Fruticultura, trabalhando na Estação Experimental de Citricultura de Bebedouro, Caixa Postal 74, 14700-000, Bebedouro (SP) <stuchi@cnpmf.embrapa.br> (17) 3344-8844.

${ }^{3}$ Estação Experimental de Citricultura de Bebedouro, Caixa Postal 74, 14700-000, Bebedouro (SP) <eecb@estacaoexperimental.com.br> (17) 3344-8844.

${ }^{4}$ Prof Titular Departamento de Ciências Exatas, Faculdade de Ciências Agrárias e Veterinárias - UNESP, Jaboticabal, (SP) <perecin@ @ fcav.unesp.br> (16) 3209-2624.
} 
TABELA 1 - Valores médios de produtividade de laranjeira 'Pêra' enxertada sobre 16 porta-enxertos, no período de 1996 a 1999 e médias das safras. Bebedouro-SP.

\begin{tabular}{|c|c|c|c|c|c|}
\hline Porta-enxertos/Anos (Cultivar) & 1996 & 1997 & 1998 & 1999 & Médias \\
\hline & \multicolumn{5}{|c|}{ kg.planta ${ }^{-1}$} \\
\hline 'Cravo’x 'Cleópatra' & $10,0 \mathrm{abcd}^{1}$ & 45,4 abc & $30,9 \mathrm{ab}$ & $52,2 \mathrm{a}$ & $34,6 \mathrm{a}$ \\
\hline Citrandarin & $16,4 \mathrm{ab}$ & $50,9 \mathrm{ab}$ & $19,6 \mathrm{ab}$ & $49,6 \mathrm{a}$ & $34,1 \mathrm{a}$ \\
\hline Pectinífera/Shekwasha & $6,3 \mathrm{~cd}$ & $53,5 \mathrm{a}$ & $43,7 \mathrm{a}$ & $49,4 \mathrm{a}$ & $31.3 \mathrm{a}$ \\
\hline 'Cravo' & $9,3 \mathrm{bcd}$ & 44,5 abc & $23,5 \mathrm{ab}$ & $46,0 \mathrm{a}$ & $30,8 \mathrm{a}$ \\
\hline Citrangor & 10,6 abcd & $27,1 \mathrm{abc}$ & $24,3 \mathrm{ab}$ & 56,2 a & $30,0 \mathrm{ab}$ \\
\hline Heen Naran & $19,7 \mathrm{a}$ & 28,9 abc & $13,6 \mathrm{~b}$ & 54,6 a & $29,2 \mathrm{ab}$ \\
\hline 'Sunki' & $14,4 \mathrm{abc}$ & 44,4 abc & $16,1 \mathrm{~b}$ & $39,9 \mathrm{a}$ & $28,7 \mathrm{ab}$ \\
\hline 'Suen-Kat' & $11,4 \mathrm{abcd}$ & 29,9 abc & $23,7 \mathrm{ab}$ & $61,5 \mathrm{a}$ & $28,1 \mathrm{ab}$ \\
\hline 'Batangas' & $10,5 \mathrm{abcd}$ & 37,9 abc & $13,9 \mathrm{~b}$ & $40,2 \mathrm{a}$ & $25,6 \mathrm{ab}$ \\
\hline 'Cleópatra' & $6,4 \mathrm{~cd}$ & $47,6 \mathrm{abc}$ & $15,5 \mathrm{~b}$ & $35,8 \mathrm{a}$ & $24,1 \mathrm{ab}$ \\
\hline Shekwasha & 7,9 bcd & $23,4 \mathrm{abc}$ & $15,5 \mathrm{~b}$ & $44,5 \mathrm{a}$ & $20,9 \mathrm{ab}$ \\
\hline Venezuela & $6,3 \mathrm{~cd}$ & $15,6 \mathrm{bc}$ & $18,1 \mathrm{ab}$ & $50,5 \mathrm{a}$ & $20,1 \mathrm{ab}$ \\
\hline Pectinífera & $13,7 \mathrm{abc}$ & $23,9 \mathrm{abc}$ & $21,1 \mathrm{ab}$ & - & $19,4 \mathrm{ab}$ \\
\hline 'Sun Chu Sha Kat' & 7,7 bcd & $32,6 \mathrm{abc}$ & $16,3 \mathrm{~b}$ & $15,4 \mathrm{a}$ & $18,3 \mathrm{ab}$ \\
\hline ‘Oneco’' & $10,0 \mathrm{abcd}$ & 29,3 abc & $10,6 \mathrm{~b}$ & $30,6 \mathrm{a}$ & $18,1 \mathrm{ab}$ \\
\hline Nasnaran & $2,2 \mathrm{~d}$ & $10,4 \mathrm{c}$ & $23,6 \mathrm{ab}$ & $23,5 \mathrm{a}$ & $13,2 \mathrm{~b}$ \\
\hline Médias & $10,2 \mathrm{D}$ & $33,3 \mathrm{~B}$ & $19,5 \mathrm{C}$ & $44,1 \mathrm{~A}$ & - \\
\hline CV (\%) & 31,5 & 35,3 & 38,9 & 44,9 & 44,2 \\
\hline
\end{tabular}

${ }^{1}$ Médias seguidas pelas mesmas letras minúsculas (maiúsculas) na coluna (linha) não diferem pelo teste de Tukey-Kramer (5\%)

conduzido sem irrigação. O delineamento experimental foi em blocos ao acaso, duas plantas por parcela, três repetições e 16 tratamentos (portaenxertos). As cultivares de porta-enxertos, cujas sementes foram obtidas de plantas existentes na EECB e no BAG do Centro APTA Citros "Sylvio Moreira", estas últimas estão identificadas pelos seus números de acesso no referido BAG, após o nome científico, são as seguintes: tangerineira 'Sun Chu Sha Kat'(Citrus reticulata Blanco ), tangerineira 'Pectinífera' (C. reticulata) - 210, 'Shekwasha' (C. depressa Hayata) 187, tangerineira 'Pectinífera/Shekwasha' (C. depressa Hayata) - 642, tangerineira 'Batangas' $(C$. reticulata $)$, tangerineira 'Oneco' $(C$. reticulata), citrangor [citrange (Poncirus trifoliata Raf. $\mathrm{x} C$. sinensis) $\mathrm{x}$ C. sinensis] - 156, citrandarin [ C.sunki hort. ex Tanaka) x Poncirus trifoliata (L.) Raf. cv. English - 256, tangerineira 'Sunki' (C. sunki), tangerineira 'Suen-Kat' (C. sunki) - 418, tangerineira 'Nasnaran' ( $C$. amblycarpa Ochse) - 812, tangerineira 'Venezuela' (C. reticulata) - 747, tangerineira 'Heen Naran' (C. lycopersicaeformis hort. ex Tan.) - 1505, limoeiro 'Cravo' (C. limonia Osbeck) x tangerineira 'Cleópatra' (C. reshni hort ex Tanaka), limoeiro 'Cravo' ( $C$. limonia), tangerineira 'Cleópatra' (C. reshni). As avaliações de produção foram realizadas nos anos de 1996 a 1999. A colheita dos frutos e pesagem foram efetuadas por parcela, calculando-se posteriormente a Produtividade média, em kg.planta ${ }^{-1}$, de cada ano e do período estudado. Os resultados obtidos foram submetidos à análise de variância, considerando o delineamento de parcelas subdivididas no tempo. As médias obtidas, nos anos de 1996 a 1999, e a média geral do experimento foram comparadas pelo teste de TukeyKramer, ao nível de $5 \%$ de probabilidade, devido à ocorrência de parcelas perdidas, uma vez que, após as avaliações de CVC, nos anos de 1995 e 1997, foram realizadas podas para a redução de inóculo e a eliminação de árvores com grau 3 de contaminação pela doença. Estas eliminações causaram a perda de parcelas em diversos tratamentos, a partir de 1997. Para isso, utilizou-se o pacote estatístico SAS (Statistical Analysis System). A qualidade dos frutos foi avaliada em 8 de julho de 1998 e em 28 de julho de 1999. Em cada avaliação, coletaram-se amostras de cinco frutos por parcela, evitando-se os frutos de ramos atacados pela CVC. Com estas amostras, determinaram-se as seguintes características de qualidade dos frutos: massa, diâmetro e altura; e do suco: sólidos solúveis totais (SST), determinado com refratômetro de leitura direta a $20^{\circ} \mathrm{C} \mathrm{e}$ expressa em porcentagem $(\mathrm{m} / \mathrm{v})$; acidez por titulação com hidróxido de sódio $0,3125 \mathrm{~N}$, expressa em g de ácido cítrico/100 $\mathrm{mL}$; ratio, que é a razão aritmética entre os sólidos solúveis totais e a acidez, rendimento em suco (RS) expresso em porcentagem $(\mathrm{m} / \mathrm{m})$ e o índice tecnológico (IT), expresso em $\mathrm{kg}$ de sólidos solúveis totais por caixa ( $\mathrm{kg}$ SST . caixa
${ }^{1}$ ) e calculado pela expressão: IT $=\left[\operatorname{RS} \times\right.$ SST x 40,8] x $10.000^{-1}$, onde o valor de 40,8 kg corresponde à da caixa-padrão industrial de citros. Para cada uma das características estudadas, calculou-se a média das duas avaliações. Os resultados obtidos foram submetidos à análise de variância, considerando o delineamento de parcelas subdivididas no tempo, e as médias foram comparadas pelo teste de Tukey-Kramer, conforme descrito anteriormente. Em razão da ocorrência da CVC no pomar experimental e com o objetivo de avaliar a influência dos portaenxertos na evolução e intensidade da doença, foram determinadas a incidência e a severidade da mesma, no segundo semestre dos anos de 1995, 1997 e 2000. A incidência foi avaliada por meio de contagem das plantas com sintomas, e posterior cálculo do percentual de plantas atacadas. A severidade foi avaliada de acordo com uma escala de notas 0 (sem sintomas) a 3 (mais de $50 \%$ dos ramos com sintomas) proposta por Salva et al. (1995). Com estes dados, calculou-se o percentual de plantas com grau máximo (3) em cada ano. Os resultados obtidos não foram analisados estatisticamente.

A Tabela 1 mostra os valores de produtividade para o período de 1996 a 1999 e a produtividade média. O experimento apresentou produtividade média de frutos baixa, $20 \%$ menor que a produtividade média das quatro primeiras safras de laranjeiras 'Pêra' clone IAC enxertadas em 'Cleópatra', conforme relataram Donadio et al. (2002). A produtividade média, em 1997, foi maior que a de 1998. Os porta-enxertos tiveram desempenhos distintos em cada ano, sugerindo uma forte influência de fatores climáticos. Os porta-enxertos, aparentemente menos afetados pelo déficit hídrico, ocorrido no período de florescimento e pegamento dos frutos em 1997, foram Pectinífera/Shekwasha, 'SuenKat', citrangor, 'Venezuela' e 'Nasnaran', pois apresentaram, em 1998, produtividade de frutos similar ou ligeiramente inferior às de 1997. Na média das quatro safras estudadas, os porta-enxertos 'Cravo' $\mathrm{x}$ 'Cleópatra', citrandarin, Pectinífera / 'Shekwasha' e 'Cravo' induziram maior produtividade, diferindo significativamente de 'Nasnaran'. Os demais porta-enxertos apresentaram valores intermediários. Não se observou uma relação entre o percentual de plantas com grau máximo de CVC em 1999 (Tabela 3) e a produtividade média de frutos, para o período 1996-1999. Talvez, isso possa ser atribuído a maiores rendimentos proporcionados por plantas com menor severidade da doença, que existiam nas parcelas. Entretanto, como as colheitas foram realizadas por parcelas, as avaliações de severidade da doença não foram efetuadas todos os anos e não houve separação dos frutos por tamanho, não possível determinar a redução na produção pela porcentagem de frutos pequenos nem pela percentagem estimada de danos conforme proposto 
TABELA 2 - Valores médios de algumas características de qualidade dos frutos de laranjeira 'Pêra' enxertada sobre 16 porta-enxertos, média das safras 1998-1999. Bebedouro-SP.

\begin{tabular}{|c|c|c|c|c|c|c|c|c|}
\hline $\begin{array}{l}\text { Porta-enxertos } \\
\text { (Cultivar) }\end{array}$ & Massa (g) & $\begin{array}{l}\text { Diâmetro } \\
(\mathrm{cm})\end{array}$ & $\begin{array}{c}\text { Altura } \\
(\mathrm{cm})\end{array}$ & $\begin{array}{c}\text { SST } \\
\left({ }^{\mathrm{o}} \text { Brix }\right)\end{array}$ & $\begin{array}{c}\text { Acidez } \\
(\%)\end{array}$ & ratio & $\begin{array}{c}\text { Suco } \\
(\%)\end{array}$ & $\begin{array}{c}\text { IT } \\
\left(\mathrm{kg} \mathrm{SST} . \text { caixa } 40.8^{-1}\right)\end{array}$ \\
\hline Cravo' x Cleópatra & $227 \mathrm{a}$ & $7,2 \mathrm{a}$ & $7,8 \mathrm{a}$ & $8,8 \mathrm{~b}$ & $0,62 \mathrm{de}$ & $14,2 \mathrm{ab}$ & $64,6 \mathrm{a}$ & 2,33 \\
\hline Sunki & $208 \mathrm{ab}$ & $7,0 \mathrm{ab}$ & $7,5 \mathrm{ab}$ & $9,2 \mathrm{ab}$ & 0,67 cde & $13,9 \mathrm{ab}$ & $62,7 \mathrm{ab}$ & 2,35 \\
\hline Cravo & $206 a b c$ & $7,0 \mathrm{ab}$ & $7,5 \mathrm{ab}$ & $8,5 \mathrm{~b}$ & $0,55 \mathrm{e}$ & $15,6 \mathrm{a}$ & $63,2 \mathrm{ab}$ & 2,20 \\
\hline Cleópatra & $203 a b c$ & $6,9 a b c$ & $7,4 \mathrm{ab}$ & $9,8 \mathrm{ab}$ & 0,70 cde & $14,1 \mathrm{ab}$ & $63,1 \mathrm{ab}$ & 2,52 \\
\hline Citrandarin & $201 \mathrm{abc}$ & $6,9 a b c$ & $7,3 \mathrm{ab}$ & $9,5 \mathrm{ab}$ & 0,71 cde & $13,3 \mathrm{abc}$ & $64,0 \mathrm{a}$ & 2,47 \\
\hline Citrangor & $195 \mathrm{abc}$ & $6,7 a b c$ & $7,5 \mathrm{ab}$ & $9,3 \mathrm{ab}$ & $0,76 \mathrm{bcd}$ & $12,2 \mathrm{abc}$ & $62,0 \mathrm{ab}$ & 2,35 \\
\hline Suen-Kat & $195 \mathrm{abc}$ & $6,9 a b c$ & $7,5 \mathrm{ab}$ & $9,3 \mathrm{ab}$ & $0,79 \mathrm{bcd}$ & $11,8 \mathrm{bc}$ & $62,0 \mathrm{ab}$ & 2,35 \\
\hline Pectinífera/Shekwasha & $192 \mathrm{abc}$ & $6,7 a b c$ & $7,4 a b$ & $9,9 \mathrm{ab}$ & 0,71 cde & $14,09 \mathrm{ab}$ & $61,4 \mathrm{ab}$ & 2,49 \\
\hline Heen Naran & $191 \mathrm{abc}$ & $6,8 \mathrm{abc}$ & $7,4 \mathrm{ab}$ & $9,7 \mathrm{ab}$ & $0,83 \mathrm{bcd}$ & $11,7 \mathrm{bc}$ & $60,2 \mathrm{ab}$ & 2,38 \\
\hline Venezuela & $191 \mathrm{abc}$ & $6,7 \mathrm{abc}$ & $7,4 a b$ & $10,5 \mathrm{ab}$ & $0,84 \mathrm{bc}$ & $12,6 \mathrm{abc}$ & $60,2 \mathrm{ab}$ & 2,58 \\
\hline Sun Chu Sha Kat & $188 \mathrm{bc}$ & $6,8 a b c$ & $7,3 \mathrm{ab}$ & $9,4 \mathrm{ab}$ & 0,72 cde & $13,3 \mathrm{abc}$ & $59,0 \mathrm{ab}$ & 2,27 \\
\hline Pectinífera & $186 \mathrm{bc}$ & $6,6 \mathrm{bc}$ & $7,4 a b$ & $9,6 \mathrm{ab}$ & $0,78 \mathrm{bcd}$ & $12,5 \mathrm{abc}$ & $60,0 \mathrm{ab}$ & 2,35 \\
\hline Batangas & $183 \mathrm{bc}$ & $6,6 \mathrm{bc}$ & $7,2 \mathrm{ab}$ & $10,1 \mathrm{ab}$ & $0,94 \mathrm{ab}$ & $11,0 \mathrm{bc}$ & $59,4 \mathrm{ab}$ & 2,43 \\
\hline Oneco & $175 \mathrm{bc}$ & $6,6 \mathrm{bc}$ & $7,2 \mathrm{ab}$ & $9,7 \mathrm{ab}$ & $0,85 \mathrm{bc}$ & $11,6 b c$ & $58,4 \mathrm{ab}$ & 2,32 \\
\hline Shekwasha & $174 \mathrm{bc}$ & $6,5 \mathrm{bc}$ & $7,3 \mathrm{ab}$ & $10,0 \mathrm{ab}$ & $0,83 \mathrm{bcd}$ & $12,3 \mathrm{abc}$ & $59,0 \mathrm{ab}$ & 2,41 \\
\hline Nasnaran & $169 \mathrm{c}$ & $6,4 \mathrm{c}$ & $7,1 \mathrm{~b}$ & $11,3 \mathrm{ab}$ & $1,13 \mathrm{a}$ & $10,0 \mathrm{c}$ & $56,9 \mathrm{~b}$ & 2,65 \\
\hline CV (\%) & 8,6 & 3,1 & 3,7 & 9,4 & 9,8 & 9,7 & 4,8 & 11,2 \\
\hline
\end{tabular}

${ }^{1}$ Médias seguidas pelas mesmas letras minúsculas não diferem pelo teste de Tukey-Kramer (5\%)

por Laranjeira \& Palazzo (1999) e por Laranjeira \& Pompeu Júnior (2002). Segundo estes últimos autores, a avaliação da reação à CVC não deve ser feita apenas com base em sintomas foliares, sendo necessária a avaliação dos danos à produção. Pelo exposto, sugere-se que, em futuros trabalhos, quando se tratar da influência dos porta-enxertos na intensidade da doença, sejam feitas avaliações que permitam estimar os danos à produção. Com exceção do IT, para todas as características de qualidade, ocorreram diferenças significativas devidas aos porta-enxertos (Tabela 2). 'Cravo' x 'Cleópatra' proporcionou maior tamanho médio dos frutos, diferindo de 'Sun Chu Sha Kat', 'Pectinífera', 'Batangas', 'Oneco', 'Shekwasha' e 'Nasnaran'. 'Cravo' x 'Cleópatra' induziu os maiores valores médios de diâmetro e altura dos frutos, diferindo de 'Pectinífera', 'Batangas', 'Oneco', 'Shekwasha' e 'Nasnaran' quanto à primeira característica e apenas de 'Nasnaran' quanto à altura. 'Nasnaran' proporcionou os maiores valores de SST, diferindo de 'Cravo' $x$ 'Cleópatra' e de 'Cravo'. O maior valor de acidez também foi encontrado com este porta-enxerto, valor este que diferiu de todos os demais, com exceção de 'Batangas'. O valor de ratio foi mais elevado com 'Cravo', diferindo significativamente de 'Suen-Kat', 'Heen Naran', 'Oneco',
'Batangas' e 'Nasnaran', indicando que estes porta-enxertos podem proporcionar colheitas mais tardias. 'Cravo' x 'Cleópatra' e citrandarin proporcionaram os maiores rendimentos em suco, diferindo significativamente de 'Nasnaran'. Em 1995, a incidência média de CVC, no pomar experimental, foi de $28,1 \%$ (Tabela 3 ). Em alguns tratamentos, a incidência foi consideravelmente maior, $50 \%$ para as cultivares SuenKat, Cravo x Cleópatra e Oneco, e 66,7\% para Pectinífera/Shekwasha. Não havia plantas com o grau máximo da doença. Na avaliação seguinte (1997), a incidência média foi de 56,3\% e detectaram-se plantas em todos os porta-enxertos com o grau máximo da doença, exceto no citrandarin. Em 1999, a incidência foi de 100\%, havendo um percentual apreciável de plantas com o grau máximo da doença $(46,9 \%)$. Com relação ao percentual de plantas com grau máximo, em 1999, Pectinífera e Pectinífera/Shekwasha apresentaram os maiores valores, $100 \%$ e $83,3 \%$, respectivamente. As menores incidências foram detectadas nos tratamentos citrangor e 'Sunki', 16,7\% para ambos, e citrandarin que não apresentou, novamente, plantas com grau máximo da doença. Os resultados obtidos sugerem que a intensidade da CVC é distinta para uma mesma variedade em função do porta-enxerto, o que diverge dos resultados relatados por Laranjeira

TABELA 3 - Incidência e severidade (plantas com grau máximo) da CVC em plantas de 'Pêra' sobre 16 porta-enxertos nos anos de 1995,1997 e 1999. Bebedouro-SP.

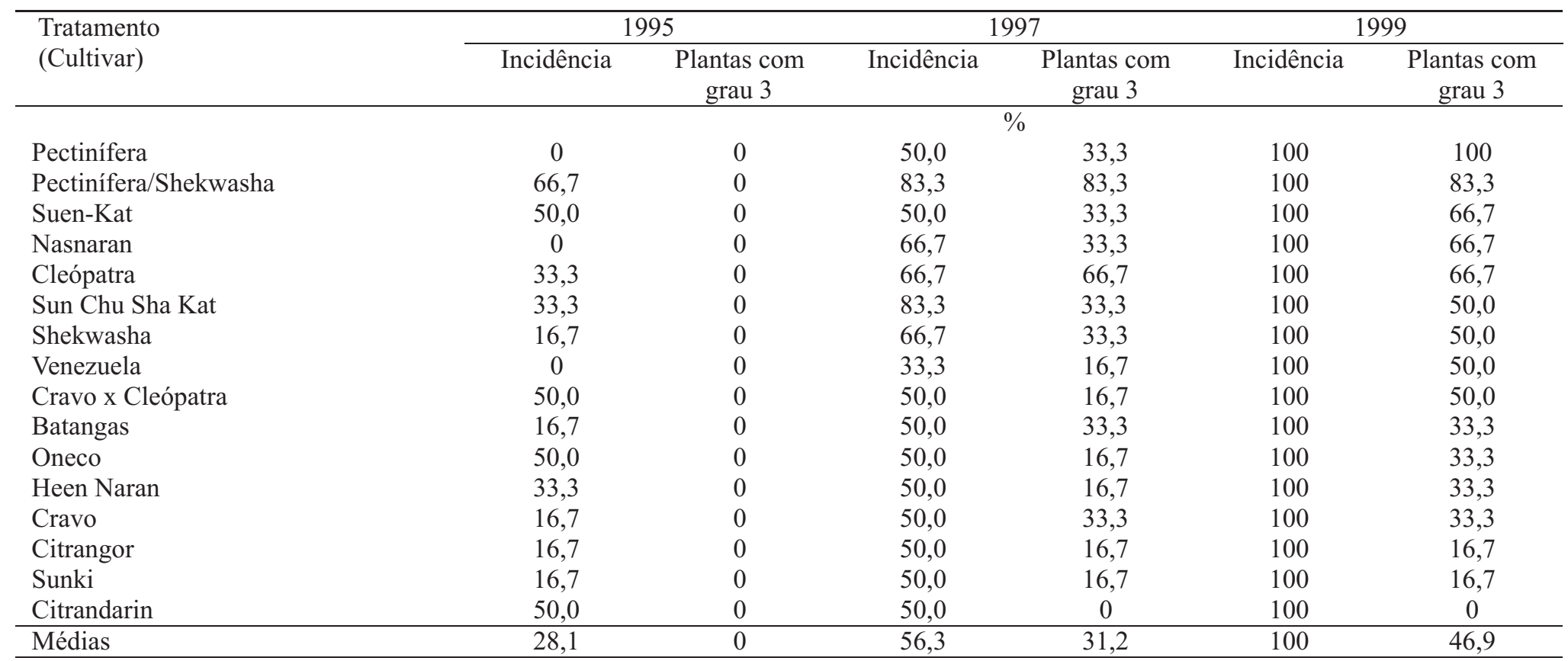


et al. (2001) e por de Stuchi et al. (1998) e está em concordância com os resultados obtidos por Stuchi et al. (2002), que empregaram o mesmo critério de avaliação adotado no presente trabalho. Os porta-enxertos, com exceção da tangerineira 'Nasnaran', induziram à laranjeira 'Pêra' qualidade e produtividades iniciais de frutos similares às do limoeiro 'Cravo'.

\section{REFERÊNCIASBIBLIOGRÁFICAS}

DONADIO, L.C; STUCHI, E.S.; SEMPIONATO, O.R. Variação de produtividade em 'Pêra' sobre 'Cleópatra' em diversos espaçamentos, sem irrigação. In: CONGRESSO BRASILEIRO DE FRUTICULTURA, 17., 2002, Belém. Anais... Belém: SBF. CD-ROM.

LARANJEIRA, F.F; POMPEU JÚNIOR, J. Comportamento de quinze cultivares de laranja-doce afetadas pela clorose variegada dos citros. Laranja, Cordeirópolis, v.23, n.2, p.401-411, 2002.

LARANJEIRA, F.F.; MÜLLER, GW.; VAZFILHO, D.; POMPEU JÚNIOR, J. Porta-enxertos não influenciam na expressão da clorose variegada dos citros (CVC) em laranjas-doces. Revista Brasileira de Fruticultura, Jaboticabal, v.23, n.1, p.212-214, 2001.

LARANJEIRA, F.F.; PALAZZO, D. Danos qualitativos à produção de laranja 'Natal' causados pela clorose variegada dos citros. Laranja, Cordeirópolis, v.20, n.1, p.77-91, 1999.

MÜLLER, G.W.; NEGRI, J.D.; MACHADO, M.A. Morte súbita dos citros (MSC): um novo desafio. Jornal de Limeira, Limeira, n.19, p.16,
2002. Ed. especial

POMPEU JÚNIOR, J. Rootstocks and scions in citriculture of the São Paulo State. In: INTERNATIONAL CONGRESS OF CITRUS NURSERYMEN, 6., 2001, Ribeirão Preto Proceedings... Bebedouro: Estação Experimental de Citricultura, 2001. p.75-82.

POMPEU JÚNIOR, J. Porta-enxertos. In: RODRIGUEZ, O.; VIEGAS, F.C.; POMPEU JÚNIOR, J.; AMARO, A.A. (Ed.) Citricultura Brasileira 2.ed. Campinas: Fundação Cargill, 1991. v. 1, p. 265-80.

SALVA, R.A. Citrus tree production in Brazil. In: INTERNATIONAL CONGRESS OF CITRUS NURSERYMEN, 6., 2001, Ribeirão Preto Proceedings... Bebedouro: Estação Experimental de Citricultura, 2001.p.11-16.

SALVA, R.A.; ROBERTO, S.R.; CARLOS, E.F. Situação da clorose variegada dos citros no Estado de São Paulo. Laranja, Cordeirópolis, v.16, n.2, p.155-164, 1995.

STUCHI, E.S.; DONADIO, L.C.; SEMPIONATO, L.C. Qualidade industrial e produção de frutos de laranjeira 'Valência' enxertada em sete porta-enxertos. Laranja, Cordeirópolis, v.23, n.2, p.453-471, 2002.

STUCHI, E.S.; DONADIO, L.C.; SEMPIONATO, L.C.; CYRILLO, F.L.L.; SILVA, J.A.A.; SOUZA, P.S. Intensidade da clorose variegada dos citros nas variedades 'Pêra' 'Homosassa' e 'Moro' (Citrus sinensis) enxertadas sobre 16 porta-enxertos em Bebedouro-SP. In: CONGRESSO PAULISTADE FITOPATOLOGIA, 21, 1998., Botucatu. Resumos... 1998. p.49. 